\title{
Genetic variant in the promoter region of microRNA-137 reduces the warfarin maintenance dose in patients with atrial fibrillation
}

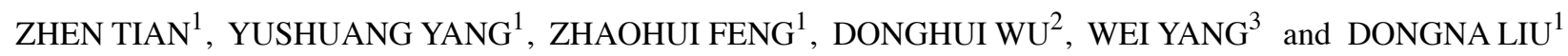 \\ ${ }^{1}$ Department of Cardiology, China-Japan Union Hospital of Jilin University; ${ }^{2}$ Department of Orthopedics, \\ People's Hospital of Jilin Province; ${ }^{3}$ Department of Psychology, Changchun Sixth Hospital, \\ Changchun, Jilin 130033, P.R. China
}

Received January 31, 2018; Accepted November 11, 2018

DOI: $10.3892 / \mathrm{mmr} .2019 .10205$

\begin{abstract}
A substantial body of research has confirmed that vitamin $\mathrm{K}$ epoxide reductase complex subunit 1 (VKORC1) plays a role in contributing to the high interpatient variability in the warfarin maintenance dose. The aim of the present study was to examine the impact of SNPs of miR-137 on the warfarin maintenance dose. Computational analysis and luciferase assay were used to search the targets of miR-137, and luciferase assay was also used to confirm the effect of the polymorphisms on the transcription of the promoter. The regulatory relationship between miR-137 and VKORC1 was detected using real-time PCR. We then performed statistical analysis to find the warfarin maintenance dose in the different groups. A total of 155 subjects were enrolled in our research, and the characteristics of the patients were collected. Using computational analysis, we identified that miR-137 binds to the VKORC1 3'untranslated region (3'UTR) and regulates the expression of VKORC1. This hypothesis was confirmed by luciferase reporter assay as miR-137 significantly reduced the VKORC1 3'UTR luciferase activity, while the luciferase activity of mutant VKORC1 3'UTR was similar to the scramble control. According to the result of the luciferase reporter assay, we found that miR-137 SNP with the presence of the A allele apparently reduced the luciferase activity. Using real-time PCR, we revealed that miR-137 negatively regulated the expression of VKORC1 in a concentration-dependent manner in liver cells. Furthermore, no difference was noted regarding the warfarin maintenance dose between the different age or gender groups, and furthermore AC + AA carriers showed a markedly higher warfarin maintenance dose than $\mathrm{CC}$ carriers. These findings collectively provide support that VKORCI is a direct target of miR-137 and the miR-137 rs2660304
\end{abstract}

Correspondence to: Professor Yushuang Yang, Department of Cardiology, China-Japan Union Hospital of Jilin University, 126 Xiantai Street, Changchun, Jilin 130033, P.R. China

E-mail: vkorc1@126.com

Key words: genetic variant, VKORC1, promoter region, microRNA-137, warfarin maintenance dose, atrial fibrillation polymorphism is associated with warfarin maintenance dose in patients with atrial fibrillation. The rs 2660304 polymorphism is a potential biomarker for predicting the clinical efficacy of warfarin in these patients.

\section{Introduction}

Warfarin is an anticoagulant widely used for preventing thromboembolic events correlated with arterial and venous thrombosis, particularly in the management of atrial fibrillation (AF). The conventional clinical treatment strategy, for example, experience-based dosing, may frequently cause higher or lower dosages of warfarin than necessary as warfarin has a limited therapeutic range and displays wide variable responses in individuals. A high dose may elevate the risk of bleeding in the initiation stage of the therapy while a low dose may not produce adequate anticoagulation (1). Hence, it is extremely important to administer the right dosage of warfarin in the initial stage for patients with AF.

Clinically, patients who receive implantation with a mechanical valve prosthesis have to be on anticoagulants such as warfarin throughout their lifetime (2). Warfarin is a widely used anticoagulant, but its therapeutic safety window is small and there are variations in the dosage for individuals. One challenge for physicians and surgeons is to determine the appropriate individual dose of warfarin; for example, a dose adequate to produce the favorable anti-clotting effect without causing adverse drug complications (3). At present, available data indicate that the initial dosage of warfarin dosing in individuals are associated with a variety of factors, such as heredity, weight, race, diet, gender, concomitant drug interactions, the presence of comorbidities, body size and age (4).

As a group of widely distributed non-coding small RNAs, microRNAs (miRNAs) consist of approximately 19 to 22 nucleotides. miRNAs target the 3'untranslated regions (UTRs) of mRNAs to inhibit the translation of genes post-transcriptionally mainly through incomplete base-pairing in mammalian cells, leading to reduced expression level of target genes (5). miRNAs are involved in the regulation of cell differentiation, growth, stress and numerous other biological processes (6-8).

DNA variations including single nucleotide polymorphisms (SNPs) may be harbored in microRNA target sites present in mRNAs, which are short substitutions, deletions 
or nucleotide insertions, with an incidence of approximately $1 \%$ in a population (9). miRNA binding to target mRNAs and subsequent gene modulation can be impacted by SNPs in miRNA target sites (10-12). In a similar manner, SNPs that impact miRNA binding may also be harbored by miRNA target sites on circRNA genes.

VKORC1 has been reported to be functionally involved in the bioactivation of vitamin $\mathrm{K}$, a key element in the coagulation system (13). It was found that VKORC1 is a target of miR-137 by using an online miRNA database. One polymorphism in the promoter region of miR-137 is believed to be able to compromise the transcription of the miRNA and reduce its expression level (14). In the present study, the miR-137/VKORC1 relationship in HepG2 cells was verified and the association between miR-137 promoter rs 2660304 polymorphism and the post-operative warfarin maintenance dose was tested.

\section{Materials and methods}

Subjects. A total of 155 Han Chinese patients with AF were enrolled in the present study between September 2016 and May 2017 at the China-Japan Union Hospital of Jilin University. The study was conducted according to the Declaration of Helsinki. All participants were diagnosed with AF, and also received long-term therapy with warfarin for at least 3 months. Data related to the demographic characteristics of the subjects including age, sex, height, weight, warfarin dose and international normalized ratio (INR) of each patient were collected prior to our research. Written informed consent was obtained from all patients or their first-degree relatives after explanation of all the potential risks. The demographic characteristics are presented in Table I. The study was approved by The Research Ethics Committees of China-Japan Union Hospital of Jilin University (approval no. 201700347).

Cell culture and transfection. Two different liver cancer cell lines, HepG2 or HepG2.2.1.5 cells were purchased from the Cell Bank of the Chinese Academy of Sciences (Shanghai, China) and incubated in Dulbecco's modified Eagle's medium (DMEM) culture medium (Gibco; Thermo Fisher Scientific, Inc.) containing $10 \%$ fetal bovine serum (FBS) in an incubator with $5 \% \mathrm{CO}_{2}$ at $37^{\circ} \mathrm{C}$. Lipofectamine 2000 reagent (Invitrogen; Thermo Fisher Scientific, Inc.) was utilized to perform the miR-137 mimic (5'-UUAUUGCUUAAGAAU ACGCGUAG-3') transfection when the cells were grown to $70-90 \%$ confluence using Lipofectamine ${ }^{\circledR} 2000$ (Invitrogen; Thermo Fisher Scientific, Inc.) following standard protocol by the supplier (Fig. 1). The transfection medium was replaced $8 \mathrm{~h}$ after transfection. Three independent tests were carried out.

RNA isolation and real-time PCR. Forty-eight hours post-transfection, the cells were harvested, and TRIzol reagent (Invitrogen; Thermo Fisher Scientific, Inc., Waltham, MA, USA) was utilized to isolate the total RNA from the liver cells following the manufacturer's guidelines. RNase-free water (Promega, Southampton, UK) was utilized to elute the RNA and stored at $-80^{\circ} \mathrm{C}$ in a refrigerating cabinet. BioTek PowerWave XS (SSi Robotics, Tustin, CA, USA) spectrophotometer was utilized to detect the purity, concentration and content of RNA. The miRNA cDNA synthesis kit (Invitrogen; Thermo Fisher Scientific, Inc.) was utilized to synthesize the cDNA (VKORC1 and miR-137). Fast SYBR Green Master Mix (Life Technologies; Thermo Fisher Scientific, Inc.) was utilized to perform real-time PCR in a $20 \mu \mathrm{l}$ volume mix, and the protocol of the reaction was carried out under the following conditions: $50^{\circ} \mathrm{C}$ for $2 \mathrm{~min}$ (initial step), $95^{\circ} \mathrm{C}$ for $10 \mathrm{~min}$, followed by 40 cycles of $95^{\circ} \mathrm{C}$ for $3 \mathrm{sec}$ for denaturation and $60^{\circ} \mathrm{C}$ for $30 \mathrm{sec}$ for elongation/hybridization. RNU43 was served as the internal control to normalize the expression of VKORC1 mRNA and miR-137. The $2^{-\Delta \Delta C q}$ method (15) was utilized to analyze the data of real-time RT-PCR. The experiment was repeated three times.

Genotype determination by TaqMan genotyping kit. The Omega FFPE DNA kit (Omega, Shanghai, China) was utilized to purify and isolate the genomic DNA based on the manufacturer's guidelines. The TaqMan Genotyping kit (Applied Biosystems; Thermo Fisher Scientific, Inc.) was performed to carry out the genotype determination.

Vector construction (test effect of rs2660304 on transcription of the promoter). The upstream region of miR-137 was amplified based on the information collected from the UCSC genome browser (http://genome.ucsc.edu/), and the PCR product was inserted into the pGL3-Basic vector (Promega, Madison, WI, USA) to generate the wild-type promoter of miR-137. The site-directed mutagenesis was used to introduce the minor allele of the rs 2660304 polymorphism.

Vector construction (test regulatory relationship between miRNA and target). The fragment of the 3'UTR (untranslated region) of VKORC1 with the predicted miR-137 binding site was amplified through PCR, the PCR products were inserted into the pGL3-Basic vector (Promega) to yield the luciferase reporter constructs, and then and the site-directed mutagenesis was used to replace the potential binding site with the reverse and complementary sequences to generate the mutant 3 'UTR of VKORC1.

Luciferase assay. In brief, the HepG2 cells $\left(2 \times 10^{6}\right)$ were seeded in 48 -well plates. When the cells were grown to $80 \%$ confluence, Lipofecamine ${ }^{2000}$ reagent (Invitrogen; Thermo Fisher Scientific, Inc.) was utilized to co-transfect the HepG2 cells with the wild-type/mutant type vector. Approximately $0.4 \mathrm{mg}$ of firefly luciferase reporter vector with the mutant target site and wild-type and $0.02 \mathrm{mg}$ of the control plasmid containing Renilla luciferase pGL3 (Promega) were utilized in this reaction. After incubation for $12 \mathrm{~h}$, the transfection medium was replaced, and passive lysis buffer (Promega) was utilized to lyse the cells, and a 96-well plate luminometer (Berthold Detection Systems, Pforzheim, Germany) was utilized to analyze the cell lysates. The Dual-Luciferase Reporter Assay system (Promega) was then utilized to measure the activities of firefly and Renilla luciferase following the manufacturer's recommendation. Three independent experiments were performed.

Western blot analysis. Western blotting was utilized to assess the expression of VKORC1 protein in HepG2 cells. The HepG2 
Table I. Demographic and clinicopathological characteristics of the AF patients recruited in the present study.

\begin{tabular}{lc}
\hline Demographic data & Patient data \\
\hline Total number & 155 \\
Mean age (years) & $52.12 \pm 10.23$ \\
Male sex, $\mathrm{n}(\%)$ & $86(55.48)$ \\
Mean weight $(\mathrm{kg})$ & $63.23 \pm 12.23$ \\
Mean height $(\mathrm{cm})$ & $165.2 \pm 18.23$ \\
Stable warfarin dose $(\mathrm{mg} /$ day $)$ & $3.08 \pm 0.24$ \\
INR & $2.28 \pm 0.55$
\end{tabular}

$\mathrm{AF}$, atrial fibrillation; INR, international normalized ratio.

A

$\begin{aligned} \text { VKORC1 } & 5^{\prime} \text {-GAGGGAAGGUCUG-AGCAAUAA-3' } \\ \text { Hsa-miR-137 } & \text { 3' -GAUGCGCAUAAGAAUUCGUUAUU- 5' } \\ \text { VKORC1 Mut } & \text { 5' -GAGGGAAGGCAUGG-CUUGCGGC-3' }\end{aligned}$

B

Human $\cdot$ AGCAAUAAAGUUUCUUAGAUCA
Chimp $\cdot$ AGCAAUAAAGUUUCUUAGAUCA
Rhesus $\cdot$ AGCAAUAAAGUUUCUUAGAUCA
Squirre $1 \cdot$ AGCAAUAAAAUUUCUUAAAUAA
Mouse $\cdot$ AACAAUAAAUUUUUCUAU----
Rat $\cdot$ AACAAUAAAUUUUUCUAU----
Rabbit $\cdot$ AGCAAUAAAAUUUCUUAACUAC
$\mathrm{Pig} \cdot$ AGCAAUAAAAUUUCUUAAAUAA
Cow $\cdot$ AGCAAUAAAAUUUCUUAAAUAA
Cat $\cdot$ AGCAAUAAAAUUUCUUAAAUAA
Dog $\cdot$ AGCAAUAAAAUUUCUUAAAUAA

Figure 1. (A) VKORC1 is a candidate target gene of miR-137 and the 'seed sequence' in the 3'UTR. (B) VKORC1 3'UTR is highly conserved among species. VKORC1, vitamin K epoxide reductase complex subunit 1; UTR, untranslated region; RLU, relative light units.

cells were harvested, and RIPA lysis buffer (Invitrogen; Thermo Fisher Scientific, Inc.) containing complete protease inhibitor mix, $0.1 \%$ sodium dodecyl sulfate (SDS), $0.5 \%$ Na-DOC, $1 \%$ NP-40, $0.05 \% \mathrm{NaN}_{3}$ and $50 \mathrm{mM}$ Tris (pH 7.4) was utilized to lyse the HepG2 cells. A BCA assay kit (Thermo Fisher Scientific, Inc.) was utilized to determine the protein concentration. Proteins ( $35 \mu \mathrm{g} / \mathrm{lane}$ ) were separated by $12 \%$ SDS-PAGE and electroblotted to polyvinylidene difluoride (PVDF) membranes (Millipore, Bedford, MA, USA). Non-fat milk (5\%) was utilized to block the membranes to avoid unspecific binding. The primary antibodies anti-VKORC1 (cat. no. ab206656; dilution 1:2,000; Abcam, Cambridge, MA, USA) and anti- $\beta$-actin (cat. no. ab8226; dilution 1:10,000; Abcam, Cambridge, MA, USA) were utilized to detect the target protein overnight at $4^{\circ} \mathrm{C}$. Horseradish peroxidase linked anti-rabbit secondary antibody (cat. no. sc-516087; dilution 1:15,000; Santa Cruz Biotechnology, Santa Cruz, CA, USA) was utilized to treat the membranes at room temperature for 60 min. ImageQuant LAS 4000 (GE Healthcare Life Sciences,
Piscataway, NJ, USA) and enhanced chemiluminescence (ECL) (PerkinElmer, Norwalk, CT, USA) were utilized to detect the signal following the manufacturer's instruction. All experiments were run in triplicate.

Statistical analysis. All results are shown as mean \pm standard deviation (SD). $\mathrm{P}<0.05$ was considered to indicate a statistically significant difference. The differences in experiments were analyzed with the use of a two-sided Student's t-test, and the significant differences between two groups were identified using the Mann-Whitney U test. Multiple variate linear analysis was performed to analyze the clinicopathological characteristics of the AF patients. SPSS 15.0 statistical package (SPSS, Inc., Chicago, IL, USA) was utilized to perform all the statistical analyses.

\section{Results}

Characteristics of the AF patients. One hundred and fifty-five participants were recruited in the present study, and the clinical data were collected at the same time. The participant baseline characteristics are summarized in Table I. The frequency of males was $55.48 \%$. The mean age was $50.12 \pm 10.23$, and the average weight was $63.23 \pm 12.23 \mathrm{~kg}$. The average height of the subjects was $165.2 \pm 18.23 \mathrm{~cm}$, the average stable warfarin dose was $3.08 \pm 0.24 \mathrm{mg} / \mathrm{day}$ and the average INR was $2.28 \pm 0.55$.

VKORC1 is a direct target gene of miR-137. Furthermore, based on the results of in silicon analysis, VKORC1 was identified as a virtual target gene of miR-137 with the binding site located at 358-365 bp of VKORC1 3'UTR (Fig. 2A). As shown in Fig. 2B, the 'seed sequence' in the VKORC1 3'UTR was highly conserved among the human, chimp, rhesus, squirrel, mouse, rat, rabbit and cow, suggested that this 'seed sequence' may play a crucial role in the progression of AF in humans.

miR-137 directly regulates VKORC1, and the interaction between VKORC1 3'UTR and miR-126 was next confirmed using luciferase assay. The wild-type 3'UTR of VKORC1 was amplified via PCR, and QuickChange XL Site-Directed Mutagenesis kit (Agilent Technologies, Inc., Santa Clara, CA, USA) was utilized to mutate the binding site of miR-137 on VKORC1 3'UTR to obtain mutant VKORC1 3'UTR. The wild-type and mutant 3'UTR of VKORC1 were inserted into the downstream of the luciferase reporter. Subsequently, for the HepG2 cells co-transfected with the luciferase conducts containing wild-type and mutant 3'UTR of VKORC1 and miR-137 mimic, the luciferase activity was detected $48 \mathrm{~h}$ post-transfection. As shown in Fig. 3, miR-137 evidently decreased the luciferase activity of the wild-type 3'UTR of VKORC1, but the miR-137 binding site mutated completely abolished the suppressive effect of miR-137 on luciferase activity. The data collectively suggested that VKORC1 is the virtual target gene of miR-137 and miR-137 suppresses the expression of VKORC1.

The rs2660304 minor allele reduces the transcription activity of the miR-137 promoter. The effect of rs2660304 on the miR-137 transcription activity of the promoter was explored using luciferase assay. As shown in Fig. 4A, the rs2660304 A allele and the rs2620304 $\mathrm{C}$ allele were respectively inserted 

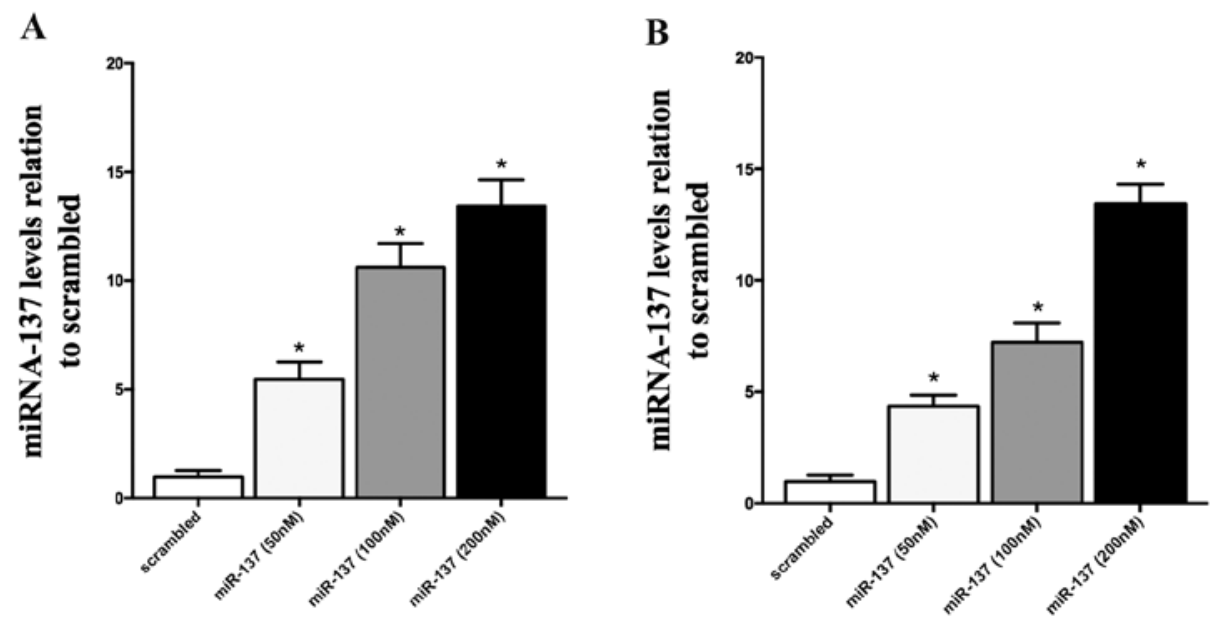

Figure 2. (A) miR-137 expression level in liver cancer cell lines HepG2 transfected with miR-137 mimic at three different concentrations. (B) miR-137 expression level in liver cancer cell lines HepG2.2.1.5 transfected with miR-137 mimic at three different concentrations. ${ }^{*} \mathrm{P}<0.05$ vs. scramble control group.

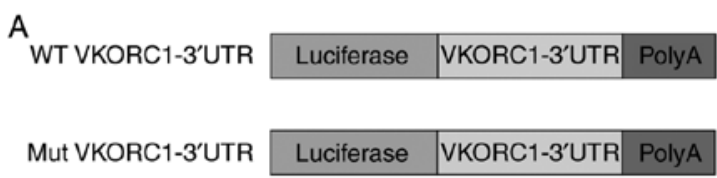

B

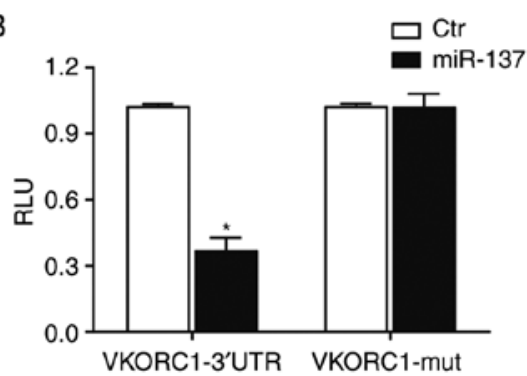

Figure 3. (A) The wild-type (WT) and mutant (Mut) 3'UTR of VKORC1 were inserted into the downstream of the luciferase reporter. (B) Luciferase activity of the HepG2 cells co-transfected with constructs with either wild-type or mutant 3'UTR of VKORC1 and miR-137 mimic or control (ctr). ${ }^{*} \mathrm{P}<0.05$ vs. control group. VKORC1, vitamin K epoxide reductase complex subunit 1; UTR, untranslated region; RLU, relative light units.

into the vector at the same site, and the above luciferase constructs were transfected into HepG2 cells. The luciferase activity was subsequently determined. As shown in Fig. 4B, it was found that the miR-137 SNP with the presence of the A allele apparently reduced the activity of luciferase when compared with miR-137, while C allele replacement with A allele in the rs 2660304 polymorphism abrogated the suppressive effect of luciferase activity, indicating that the presence of the minor allele of the polymorphism in the promotor region of miR-137 substantially reduced the transcription activity of the promoter.

miR-137 suppresses VKORC1 expression in vivo. To specifically confirm that miR-137 suppressed the expression of VKORC1 in two cell lines, HepG2 and HepG2.2.1.5 cells respectively, using Lipofectamine 2000, the cultured cells were transfected with different concentrations of miR-137 mimics (50, 100 and $200 \mathrm{nM}$; Fig. 5). A concentration of $100 \mathrm{nM}$ miR-137 mimics caused a significant decrease in the VKORC1

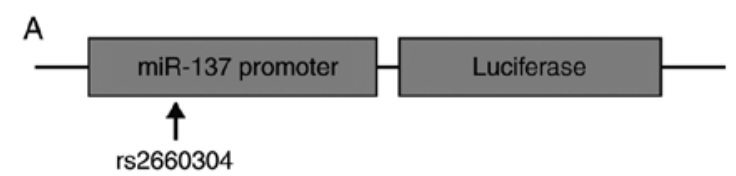

B

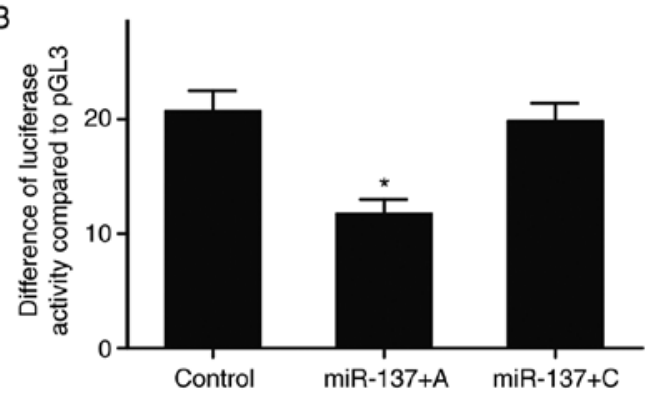

Figure 4. (A) rs2660304 polymorphism A allele and C allele cloned into the luciferase gene. (B) Effects of the different alleles on the activity of luciferase. ${ }^{*} \mathrm{P}<0.05$ vs. control group.

expression level, and $200 \mathrm{nM}$ miR-137 mimics provoked $\sim 50 \%$ decrease in VKORC1 expression related to that in the scramble control. This confirmed that miR-137 is a direct regulator of VKORC1, and miR-137 suppressed the VKORC1 expression in a concentration-dependent manner in the two culture cell lines (Fig. 5A, HepG2; Fig. 5B, HepG2.2.1.5).

Warfarin maintenance dose in the different groups. We used SPSS 15.0 statistical package (SPSS, Inc., Chicago, IL, USA) to analyze the maintenance dose in the different groups, and as shown in Fig. 6, no difference was noted regarding the warfarin maintenance dose between the different age or sex groups (Fig. 6A). Furthermore, CC carriers showed a significantly lower warfarin maintenance dose than the CA+AA carriers (Fig. 6B).

\section{Discussion}

Studies have shown a correlation between polymorphisms in the vitamin $\mathrm{K}$ epoxide reductase complex subunit 1 (VKORCl) gene and warfarin dose requirement $(16,17)$. Shomron (18), 
A

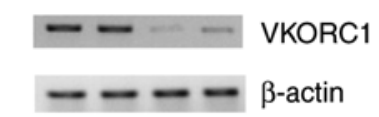

$50 \mathrm{nM}$

$100 \mathrm{nM}$

$200 \mathrm{nM}$

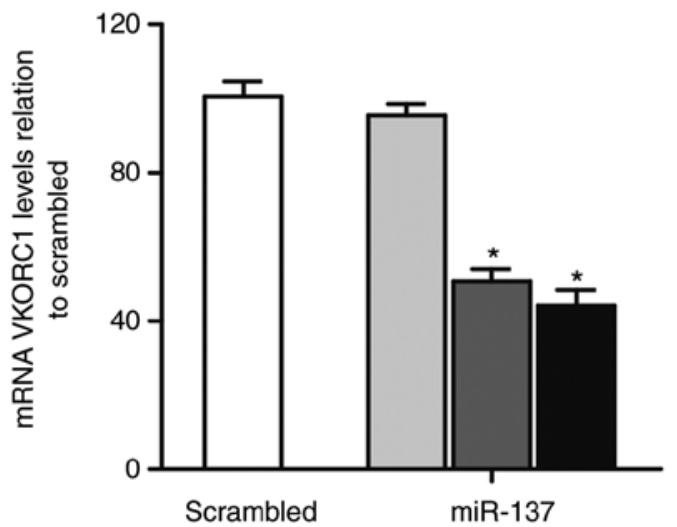

B

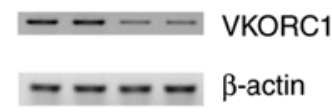

$50 \mathrm{nM}$

$100 \mathrm{nM}$

$200 \mathrm{nM}$

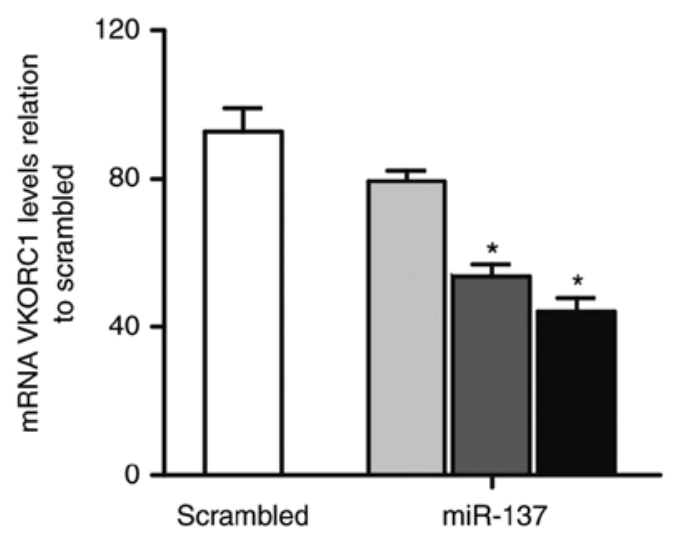

Figure 5. (A) VKORC1 expression level in liver cancer cell lines HepG2 transfected with miR-137 mimic at three different concentrations. (B) VKORC1 expression level in liver cancer cell lines HepG2.2.1.5 transfected with miR-137 mimic at three different concentrations. VKORC1, vitamin K epoxide reductase complex subunit $1 .{ }^{*} \mathrm{P}<0.05$ vs. scramble control group.
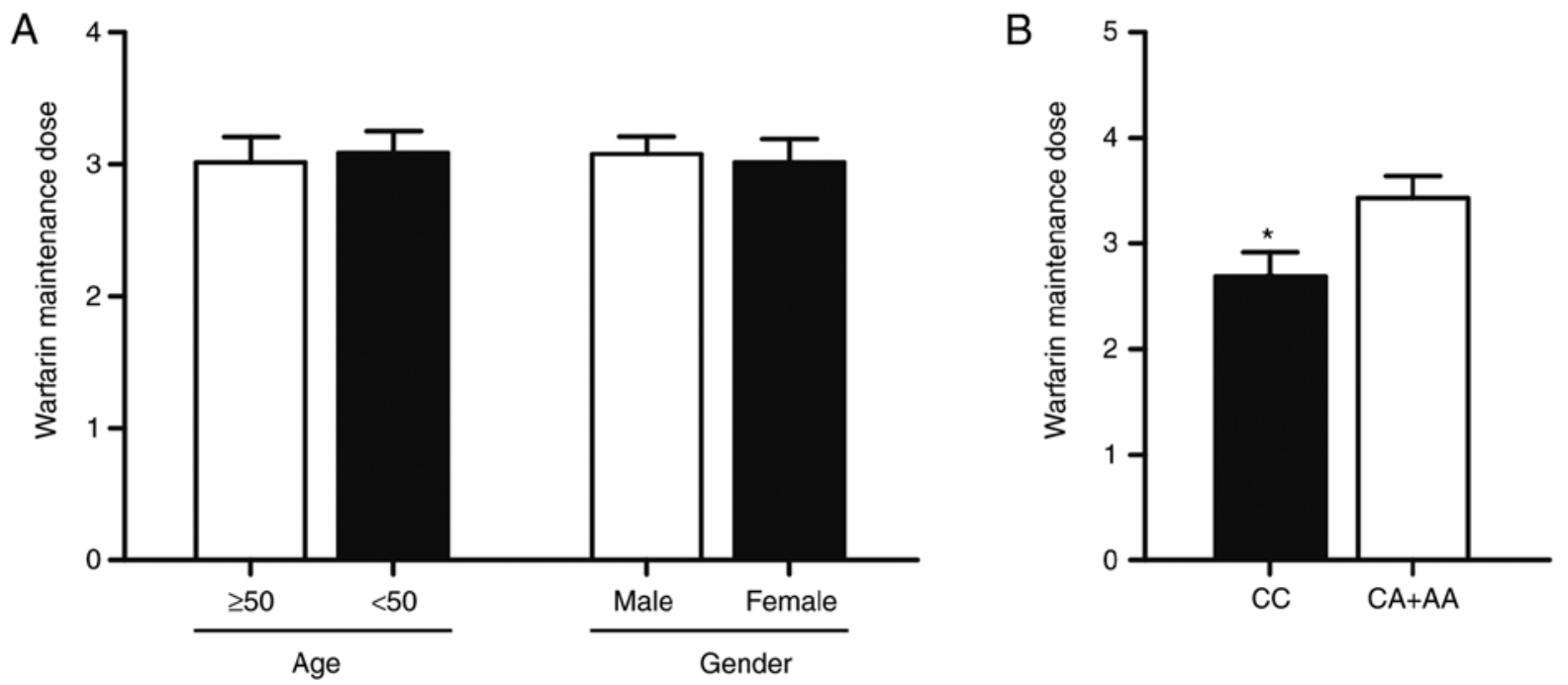

Figure 6. (A) Warfarin maintenance dose between different age group (in years). (B) Warfarin maintenance dose between $\mathrm{CC}$ and $\mathrm{CA}+\mathrm{AA}$ carriers. ${ }^{*} \mathrm{P}<0.05$ vs. CA+AA group.

discovered 2 highly evolutionarily conserved binding sites for miR-137 and miR-133 on the VKORC1 gene by applying bioinformatic tools, indicating that these miRNAs likely modulate VKORC1. A recent study by Perez-Andreu et al (19), demonstrated the possible binding sites for miR-137 and miR-133, but indicated only co-expression of miR-133 with VKORC1 in human hepatocytes. They also indicated that the VKORC1 mRNA expression was reduced by overexpression of miR-133 in a dose-dependent manner. Furthermore, in subjects who carry the $\mathrm{G}$ allele (rs9923231 in the promoter region), this modulation of VKORC1 mRNA expression was also found, which is correlated with an elevated transcription rate of VKORC1 mRNA. Hence, it is likely to predict that variants in miR-133 genes, resulting in an abnormal modulation of the VKORC1 gene, could change the effectiveness of warfarin treatment. In this study, we performed in silicon analysis, and identified that
VKORC1 is a virtual target gene of miR-137, and VKORC1 3'UTR is highly conserved among species. A luciferase assay was then conducted to verify that miR-137 directly mediates VKORC1 and the interaction between miR-137 and VKORC1, and found that miR-137 evidently decreased the luciferase activity of wild-type 3'UTR of VKORC1, while the luciferase activity of the mutant VKORC1 3'UTR was comparable with the control in the miR-137-overexpressing cells. Furthermore, it was specifically confirmed that miR-137 negatively regulated the expression of VKORC1 in the two liver cancer cell lines, and it was found that in liver cancer cells, miR-137 is a direct regulator of $\mathrm{VKORC} 1$, and miR-137 dose-dependently suppressed VKORC1 expression.

VKORC1 is known as an enzyme which is encoded by the $V K O R C 1$ gene in humans. Vitamin $\mathrm{K}$ is critical for blood clotting after being activated by enzymes (20). In some blood-clotting 
proteins, this form of vitamin $\mathrm{K}$ is activated by enzymes and is a decreased form necessary for the carboxylation of glutamic acid residues. The product of this gene encodes the enzyme that contributes to decreasing vitamin $\mathrm{K}$ 2,3-epoxide to the form being activated by enzymes. The vitamin $\mathrm{K}$ antagonist known as warfarin and deficiency of vitamin $\mathrm{K}$ can result in life-threatening bleeding, and the product of this gene exhibits sensitivity to warfarin. There is a correlation between the mutations in this gene in humans and clotting factors dependent on vitamin $\mathrm{K}$. In rats and humans, it has also been correlated with resistance to warfarin; however, such mutations are not common except in certain Jewish and Ethiopian populations. Two pseudogenes have been found on the $\mathrm{X}$ chromosome and chromosome 1 . There is also a study which has described 2 alternatively spliced transcripts which encode distinct isoforms (20).

VKOR complex, subunit 1 (VKORCl) is situated on chromosome 16 at band p11.2. VKORCl is primarily expressed in the liver; but smaller $V K O R C 1$ amounts are found in the pancreas and heart. A polymorphism within the promoter region of VKORC1, a guanine to adenine conversion at position-1639 (-1639 G>A, rs9923231), is related to a decreased requirement of warfarin in individuals of Asian descent and Caucasians. Reduced VKORC1 mRNA production by the $-1639 \mathrm{~A}$ allele and decreased enzyme VKOR expression may have led to this (21). Patients with the variant of the 1639A promoter need a lower warfarin dose due to decreased VKOR expression (22). The VKORCI-1639A allele has been shown to be present in the majority of people of Asian descent; however, is less common in individuals of African descent and Caucasian individuals by population studies. The VKORCI-1639G allele related to increased expression and activity of $\mathrm{VKORC} 1$ is predominant in individuals of African descent. Some research remains doubtful whether the-1639G allele would lead to the dosing variability of warfarin in individuals of African descent (23-25). Warfarin dosing may be affected by other alleles in VKORC1; for example, the Asp36Tyr amino acid substitution would result in a mild to moderate warfarin dose increase (26). In this study, we found that no difference was noted regarding the warfarin maintenance dose between different age or sex groups (Fig. 6A), and furthermore, AC + AA carriers showed a significantly higher warfarin maintenance dose than CC carriers (Fig. 6B). Furthermore, the effect of rs2660304 on the miR-137 transcription activity of the promoter was investigated using luciferase assay. The rs2660304 polymorphism A allele and rs2660304 polymorphism $\mathrm{C}$ allele were inserted into the luciferase gene, and it was found that miR-137 SNP with the presence of the A allele substantially suppressed the activity of luciferase when compared with miR-137, while C allele replacement with the A allele in the rs 2660304 polymorphism abrogated the suppressive impact of luciferase activity. Notably, miR-137 is not only involved in the control of the coagulation system but also has been reported to be involved in other systems such as the nervous system (27) and tumorigenesis (28), indicating a widespread and multiple system involvement of miR-137 and this polymorphism may also be associated with other function of different systems.

There are limitations in the present study. Firstly, further research with a larger sample size is needed to confirm the conclusions of this study. Secondly, further functional analysis is warranted to confirm the interaction between miRNA-137 and $V K O R C 1$.
In conclusion, these findings collectively provide support that $V K O R C 1$ is a direct target of miR-137 and the miR-137 rs2660304 polymorphism is associated with warfarin maintenance dose in the management of AF. The rs2660304 polymorphism is a potential biomarker to predict the clinical efficacy of warfarin in AF patients.

\section{Acknowledgements}

Not applicable.

\section{Funding}

No funding was received.

\section{Availability of data and materials}

The datasets used during the present study are available from the corresponding author upon reasonable request.

\section{Authors' contributions}

ZT and YSY conceived and designed the study. ZT, ZHF and DHW performed the experiments. ZHF, WY and DNL analyzed and visualized the data. WY and DNL wrote the paper. ZT, YSY, ZHF and DHW reviewed and edited the manuscript. All authors read and approved the manuscript and agreed to be accountable for all aspects of the research, ensuring that the accuracy or integrity of any part of the work are appropriately investigated and resolved.

\section{Ethics approval and consent to participate}

All experimental protocols were approved by the Research Ethics Committees of China-Japan Union Hospital of Jilin University (approval no. 201700347).

\section{Patient consent for publication}

Not applicable.

\section{Competing interests}

The authors declare that they have no competing interests.

\section{References}

1. Yoshizawa, Hayashi H, Tashiro Y, Sakawa S, Moriwaki H, Akimoto T, Doi O, Kimura M, Kawarasaki Y, Inoue $\mathrm{K}$ and Itoh K: Effect of VKORC1-1639 G>A polymorphism, body weight, age, and serum albumin alterations on warfarin response in japanese patients. Thromb Res 124: 161-166, 2009.

2. Kidane AG, Burriesci G, Cornejo P, Dooley A, Sarkar S, Bonhoeffer P, Edirisinghe M and Seifalian AM: Current developments and future prospects for heart valve replacement therapy. J Biomed Mater Res B Appl Biomater 88: 290-303, 2009.

3. Glurich I, Burmester JK and Caldwell MD: Understanding the pharmacogenetic approach to warfarin dosing. Heart Fail Rev 15: 239-248, 2010.

4. Arellano-Rodrigo E, Home monitoring of warfarin effects. N Engl J Med 364: 378-379, 2011.

5. Inui M, Martello G and Piccolo S: MicroRNA control of signal transduction. Nat Rev Mol Cell Biol 11: 252-263, 2010. 
6. Chen CZ, Li L, Lodish HF and Bartel DP: MicroRNAs modulate hematopoietic lineage differentiation. Science 303: 83-86, 2004.

7. Poy MN, Hausser J, Trajkovski M, Braun M, Collins S, Rorsman P, Zavolan M and Stoffel M: miR-375 maintains normal pancreatic alpha-and beta-cell mass. Proc Natl Acad Sci USA 106: 5813-5818, 2009.

8. Hornstein E, Mansfield JH, Yekta S, Hu JK, Harfe BD, McManus MT, Baskerville S, Bartel DP and Tabin CJ: The microRNA miR-196 acts upstream of Hoxb8 and Shh in limb development. Nature 438: 671-674, 2005.

9. Borel $\mathrm{C}$ and Antonarakis SE: Functional genetic variation of human miRNAs and phenotypic consequences. Mamm Genome 19: 503-509, 2008.

10. Saetrom P, Biesinger J, Li SM, Smith D, Thomas LF, Majzoub K, Rivas GE, Alluin J, Rossi JJ, Krontiris TG, et al: A risk variant in an miR-125b binding site in BMPR1B is associated with breast cancer pathogenesis. Cancer Res 69: 7459-7465, 2009.

11. Ryan BM, Robles AI and Harris CC: Genetic variation in microRNA networks: The implications for cancer research. Nat Rev Cancer 10: 389-402, 2010

12. Gamazon ER, Ziliak D, Im HK, LaCroix B, Park DS, Cox NJ and Huang RS: Genetic architecture of microRNA expression: Implications for the transcriptome and complex traits. Am J Hum Genet 90: 1046-1063, 2012

13. Sridharan K, Modi T, Bendkhale S, Kulkarni D, Gogtay NJ and Thatte UM: Association of genetic polymorphisms of CYP2C9 and VKORC1 with bleeding following warfarin: A case-control study. Curr Clin Pharmacol 11: 62-68, 2016.

14. Warburton A, Breen G, Bubb VJ and Quinn JP: A GWAS SNP for schizophrenia is linked to the internal MIR137 promoter and supports differential Allele-Specific expression. Schizophr Bull 42: 1003-1008, 2016.

15. Livak KJ and Schmittgen TD: Analysis of relative gene expression data using real-time quantitative PCR and the 2(-Delta Delta C(T)) method. Methods 25: 402-408, 2001.

16. Rieder MJ, Reiner AP, Gage BF, Nickerson DA, Eby CS McLeod HL, Blough DK, Thummel KE, Veenstra DL and Rettie AE: Effect of VKORC1 haplotypes on transcriptional regulation and warfarin dose. N Engl J Med 352: 2285-2293, 2005.

17. D'Andrea G, D'Ambrosio RL, Di Perna P, Chetta M, Santacroce R, Brancaccio V, Grandone E and Margaglione M: A polymorphism in the VKORC1 gene is associated with an interindividual variability in the dose-anticoagulant effect of warfarin. Blood 105: 645-649, 2005.
18. Shomron N: MicroRNAs and pharmacogenomics. Pharmacogenomics 11: 629-632, 2010.

19. Peréz-Andreu V, Teruel R, Corral J, Roldán V, García-Barberá N, Salloum-Asfar S, Gómez-Lechón MJ, Bourgeois S, Deloukas P, Wadelius M, et al: miR-133a regulates vitamin $\mathrm{K}$ 2,3-epoxide reductase complex subunit 1 (VKORC1), a key protein in the vitamin K cycle. Mol Med 18: 1466-1472, 2013.

20. Ainle FN, Mumford A, Tallon E, McCarthy D and Murphy K: A vitamin $\mathrm{K}$ epoxide reductase complex subunit 1 mutation in an Irish patient with warfarin resistance. Ir J Med Sci 177: 159-161, 2008.

21. Yuan HY, Chen JJ, Lee MT, Wung JC, Chen YF, Charng MJ, Lu MJ, Hung CR, Wei CY, Chen CH, et al: A novel functional VKORC1 promoter polymorphism is associated with inter-individual and inter-ethnic differences in warfarin sensitivity. Hum Mol Genet 14: 1745-1751, 2005.

22. Wang D, Chen H, Momary KM, Cavallari LH, Johnson JA and Sadée W: Regulatory polymorphism in vitamin K epoxide reductase complex subunit 1 (VKORC1) affects gene expression and warfarin dose requirement. Blood 112: 1013-1021, 2008.

23. Kealey C, Chen Z, Christie J, Thorn CF, Whitehead AS, Price M, Samaha FF and Kimmel SE: Warfarin and cytochrome P450 2C9 genotype: Possible ethnic variation in warfarin sensitivity. Pharmacogenomics 8: 217-225, 2007.

24. Schelleman H, Chen Z, Kealey C, Whitehead AS, Christie J, Price M, Brensinger CM, Newcomb CW, Thorn CF, Samaha FF and Kimmel SE: Warfarin response and vitamin K epoxide reductase complex 1 in African Americans and Caucasians. Clin Pharmacol Ther 81: 742-747, 2007.

25. Kimmel SE, Christie J, Kealey C, Chen Z, Price M, Thorn CF, Brensinger CM, Newcomb CW and Whitehead AS: Apolipoprotein E genotype and warfarin dosing among Caucasians and African Americans. Pharmacogenomics J 8: 53-60, 2008.

26. Loebstein R, Dvoskin I, Halkin H, Vecsler M, Lubetsky A, Rechavi G, Amariglio N, Cohen Y, Ken-Dror G, Almog S and Gak E: A coding VKORC1 Asp36Tyr polymorphism predisposes to warfarin resistance. Blood 109: 2477-2480, 2007.

27. Mahmoudi E and Cairns MJ: MiR-137: An important player in neural development and neoplastic transformation. Mol Psychiatry 22: 44-55, 2017.

28. Neault M, Mallette FA and Richard S: miR-137 modulates a tumor suppressor network-inducing senescence in pancreatic cancer cells. Cell Rep 14: 1966-1978, 2016. 